\title{
Ritagli di lingua: uno sguardo sulle carte linguistiche di Franca Brambilla Ageno all'Accademia della Crusca
}

\author{
Caterina Canneti
}

PUBBLICATO: 31 DICEMBRE 2019

$\mathrm{N}$

el corso della sua incessante attività di studio, è noto che Franca Brambilla Ageno si occupò non soltanto di filologia, ma anche di lingua. Le testimonianze di questo grande interesse si rintracciano, in particolare, tra le carte del Fondo a lei dedicato presso l'Archivio dell'Accademia della Crusca. La visione d'insieme di questo importante materiale e la sua consistenza complessiva riflettono l'impegno e la vastità d'interessi della studiosa: esaminare da vicino le carte di Franca Brambilla Ageno è un po' come entrare nel suo retrobottega e osservare la sua scrivania negli anni in cui essa stava lavorando ai suoi importanti studi. Dei molti saggi linguistici da lei pubblicati, infatti, è possibile, proprio attraverso le carte d'Archivio, ricostruire il processo di elaborazione e valutare il materiale da lei utilizzato. Questo intervento non vuole illustrare l'intera consistenza delle carte della Ageno, ma ha l'intenzione di offrire alcuni spunti di osservazione riguardo ai retroscena dei suoi lavori linguistici, al fine di illustrare, attraverso alcuni esempi, la natura dell'importantissimo materiale autografo conservato alla Crusca, in relazione alla sua vasta produzione scientifica.

Di Franca Brambilla Ageno come studiosa di lingua parla Ghino Ghinassi nell'Introduzione al volume che raccoglie i suoi Studi lessicali, uscito a stampa nel 2000, qualche anno dopo la sua scomparsa (avvenuta nel 1995):

Non servirebbero forse altre parole per introdurre il lettore in questa selva rigogliosa in cui quelli che potrebbero apparire ritagli caduti dal tavolo di un editore di testi antichi rivelano a lungo andare un'organicità e un rigore che li ricompattano in un lavoro sistematico dalle indefinite sfaccettature ${ }^{\mathrm{I}}$.

Come si può vedere, ho sottolineato alcuni termini chiave che, a mio parere, rappresentano bene non solo la consistenza delle carte del Fondo Franca Brambilla Ageno, ma anche il modo di lavorare della studiosa: selva rigogliosa, ritagli, organicitá, rigore. L'attività della Ageno, dunque, è ben testimoniata dai materiali d'Archivio: da questi documenti è certo evidente l'energia che essa ha profuso nei suoi studi e l'attenzione che ha dimostrato nella stesura dei propri appunti. Ogni carta ha una specifica collocazione, ogni scheda si riferisce a un preciso fenomeno linguistico, a una determinata citazione bibliografica, segno di quale sia stata l'operosità instancabile e la tenacia di una delle maggiori filologhe (e, in questo caso, storiche della lingua) del secolo scorso.

Nel suo necrologio per la studiosa, Carlo Delcorno ricorda la molteplicità di interessi della Ageno e illustra le sue pubblicazioni in ambito linguistico:

Gli studi propriamente linguistici dell'Ageno si svolgono lungo molteplici direttive, che qui possono essere solo accennate, senza neppure la pretesa di un elenco esaustivo: dalle molte schede lessicali pubblicate nella rivista di Bruno Migliorini, "Lingua nostra" [...], alle indagini sull'umbro antico nel Cantico di Frate Sole [...], alle ricerche sulla lingua popolaresca dei riboboli [...], sui proverbi e sulle frasi proverbiali, per le quali aveva programmato un repertorio di ampio respiro [...], alle difficili ricerche sulla semantica del gergo e del furbesco antico $[\ldots]^{2}$. 
Si parla, dunque, delle moltissime schede lessicali e degli articoli pubblicati su "Lingua nostra", degli studi sull'umbro antico, sulla lingua popolaresca, sui proverbi e sul gergo. Un ambito di indagine, quindi, che coinvolge la lingua e gli autori, soprattutto dell'ambito popolare e colloquiale, che, a detta di Delcorno, si è svolto "lungo molteplici direttive". Nel Fondo, infatti, sono numerose anche le carte con appunti e ricerche sui dialetti.

\section{Studi di lingua tra le carte}

Il Fondo Franca Brambilla Ageno, conservato all'Accademia della Crusca in due armadi dedicati, è costituito da ventuno pezzi, tra scatole e schedari, contenenti materiale di varia tipologia: bozze di stampa, appunti, riproduzioni di manoscritti, estratti, volumi, articoli e recensioni, lettere3 [I]. Tale materiale, in gran parte costituito dalle carte autografe relative ai lavori sui testi e sulla lingua, fu donato alla Crusca dalla figlia della studiosa, Elena Brambilla, il giorno zo luglio I9994.

Nel fascicolo segnato iz88 (Sacchetti I), ad esempio, molte sono le carte linguistiche prodotte dalla Ageno nel corso dei suoi lavori su Franco Sacchetti5, in particolare nel periodo in cui la studiosa stava lavorando a una nuova edizione del Trecentonovelle (mai uscita a stampa; pubblicò soltanto alcuni saggi e recensioni di edizioni precedenti ${ }^{6}$ ) e delle altre opere dell'autore (esce a stampa l'edizione delle Rime nel I9907). Nell'u.d. I8, ad esempio, si trovano vari gruppi di schede riguardanti l'uso del congiuntivo potenziale e delle forme verbali nei testi antichi (35 c cc.), la presenza di espressioni proverbiali in alcuni autori del Trecento (I6 cc.), lo studio etimologico di nomi latini terminanti in -o, -onis (77 cc.) e l'osservazione di specifici fenomeni linguistici (si tratta di ben $520 \mathrm{cc}$.). Le schede in questione hanno la stessa dimensione per ogni unità considerata (talvolta, corrispondono alla misura di un quarto di foglio $\mathrm{A}_{4}$, altre volte a un ottavo) e sono ricavate per la maggior parte dei casi da fogli di carta (alcune volte, invece, sono in cartoncino più spesso). Ciò che più colpisce di queste carte, oltre alla precisione della grafia, è la sistematicità e la metodicità nella stesura degli appunti: tutte le schede sono ordinate secondo specifici criteri (in ordine alfabetico per termine, oppure suddivise per fenomeno linguistico) e, per ogni unità considerata, esse sono tutte della stessa dimensione. Fornisco qui di seguito alcuni esempi relativi all'osservazione delle forme verbali nei testi antichi (fasc. I388, u.d. I8c):

Eziandio e propri parenti ci fa odiare, se non solo in quanto volessero Cristo - Colombini Io, 38. (c. 329)

Quanto più beio, più incendo nel cuore - Bianco 9, 26. (c. 347)

E di qua dall'Arno e di là arsono e dibruciarono ogni cosa - Velluti, 232. (c. 350)

In ogni passo la Ageno ha sottolineato la forma interessata; in ogni carta, poi, ha posto la stessa struttura del riferimento topografico (nome dell'autore, capitolo, paragrafo o riga). È interessante notare che la studiosa ha fatto ricorso per queste carte a un contenitore di fortuna, sul quale ha posto a mano (forse in tempi successivi) due diverse intestazioni sui due lati ("Schede con lessico della frottola della lingua nova" e "Varie (Schede con lessico della frottola di lingua nova)"): si tratta del contenitore di un esemplare del De Felice-Duro ${ }^{8}$, che si è trasformato, all'occorrenza, in una scatola in cui riporre le carte di lavoro [2]. Il ricorso a questo metodo di conservazione fa parte di un atteggiamento che si è rintracciato spesso nel Fondo della studiosa, come si vede per l'u.d. 2 del fasc. I4I7ter (Materiali lessicali III), contenente le schede di lavoro prodotte durante la stesura di una delle pubblicazioni più importanti, Il verbo nellitaliano antico: ricerche di sintassi (Milano-Napoli, Ricciardi, I964): in un vecchio contenitore di latta per dolciumi si trovano 26 buste, ognuna con una specifica intestazione riferita a un fenomeno morfo-sintattico o a una forma verbale e ognuna contenente 
schede di piccolo formato con esempi d'autore relativi al fenomeno in questione [3]. Considerando, ad esempio, la busta $\mathrm{H}$, con intestazione fare, lasciare, vedere + infinito, si trovano schede con i seguenti passi:

e familiarmente ricevatolo, il fece sedere - Dec. I, 3, 8. (c. I)

e sete sì piacenti ed amorosi, che vi fareste a un empero amare - Guitt., R 47, 5-6. (c. 5)

pe' quali io feci prendere in pagamento a Michele le dette due case - Velluti, I49. (c. 7)

Anche in questo caso, come nel precedente esaminato, Franca Brambilla Ageno riporta con sistematicità un esempio per scheda, sottolineando la perifrasi verbale che le interessa e indicando, con la stessa struttura ricorrente, il riferimento topografico. Le migliaia di schede presenti in questa u.d., dunque, riportano altrettanti passi d'autore che hanno costituito una base imprescindibile per l'importantissimo saggio pubblicato dalla Ageno.

Gli studi sul verbo rappresentano una buona parte della bibliografia linguistica prodotta da Franca Brambilla Ageno: dagli articoli pubblicati in rivista (Un antico costrutto concessivo, in "Lingua nostra", XI, I950, p. I8; Fare e usare modali, in "Studi di filologia italiana", XII, I954, pp. 325-333; Un antico caso di proposizione concessiva, in "Lingua nostra", 1954, pp. 6-8; L'aspetto del verbo in un tipo arcaico di proposizioni imperative e finali e Piucchepperfetto per imperfetto congiuntivo in proposizioni dipendenti, in "Studi di Filologia italiana", XVII, I959, pp. 239-274; Indicativo in dipendenza da credere e sinonimi, in "Lingua nostra", XXII, I96I pp. 6-8; Gerundio coordinato con indicativo precedente, in "Lingua nostra", XXVII, I966, p. 30; Osservazioni minime sull'uso dell'articolo determinato nella coordinazione e Presente pro futuro: due norme sintattiche dellitaliano antico, in "Studi di Grammatica italiana", IV, I975, pp. 2-27; Un esempio d'impersonale a forma attiva, in "Lingua nostra", XXXVI, I975, pp. IIO-III; Aspetti della storia della lingua: la trasmissione dei moduli sintattici e le loro modificazioni attraverso il tempo, in "Studi di Grammatica italiana", VII, I978, pp. 353-373), ai saggi in volume (Congiuntivo potenziale nell'antico italiano, in Saggi e ricerche in memoria di Ettore Li Gotti, vol. I, Palermo, G. Mori \& figli, 196I, pp. 3-24).

Sempre in relazione al verbo, si rintracciano nel Fondo alcune testimonianze dell'interesse di Franca Brambilla Ageno per la lingua di Dante: gran parte del materiale dantesco prodotto dalla studiosa, soprattutto le carte relative al lavoro per l'edizione del Convivio9, è conservato oggi presso la Società Dantesca di Firenze, anche se alcuni materiali si rintracciano nei fascicoli dell'Archivio della Crusca. Si guardino, ad esempio, le schede sul Paradiso dantesco (fasc. I4I bbis, Materiali lessicali II), contenute in una busta con intestazione: "Parad. I-IV" (u.d. 4): si tratta di II5 carte di appunti autografi riguardanti, nello specifico, alcune forme verbali presenti nei primi quattro canti del Paradiso e in altri canti della Commedia. Riporto qui di seguito un esempio di scheda:

cC. I3-I4:

33. quando alcun di sé asseta: assetare in senso proprio, 'dar sete a', 'rendere assetato', non compare nella Divina Commedia, neppure nel canto dei golosi (Purg., 23), mentre qui troviamo, appunto in senso proprio, l'unico esempio di affamare: «Già era in ammirar che sì li affama» (v. 37). Il verbo assetare è usato da Dante parecchie volte sempre nell'identica sede del verso, cioè in rima, e sempre con senso traslato. Tre volte, con questa, è unito a un complemento di limitazione: «l'anima mia gustava di quel cibo / che, saziando di sé, di sé s'asseta» (Purg., 3I, I28-I29); «d'altro non ci asseta» (Parad., 3, 72); altrove è unito a un complemento che dirà modale: «e che m'asseta / di dolce desiar» (Parad., 15, 65-66); e altrove è usato assolutamente: «la superbia ch'asseta» (Parad., I9, I2r: il complemento oggetto è taciuto). 
Anche in questo caso, le analisi proposte tra le carte della studiosa si rivelano molto significative e dimostrano di nuovo il grande interesse della Ageno per la lingua, in questo caso per quella della Commedia. Di nuovo, la studiosa sottolinea forme e perifrasi, commentandole e confrontandole con altri esempi danteschi.

Come già precedentemente visto, dunque, non mancano pubblicazioni della Ageno su Dante: dalle schede su "Lingua nostra" (Parole di Dante, XVI, 1955, pp. 40-43; 'Bestilitade': un uso dantesco, XLV, I984, p. 59; Un recupero dantesco: "prolificabile', XLIV, I983, p. 53-54), ai numerosi saggi negli "Studi Danteschi" (Per linterpretazione di quattro passi danteschi, XXXIV, I957, pp. 205-I5; Annotazioni sintattiche ad alcuni passi della «Commedia», XLIII, I965, pp. 339-360; Note a passi della Divina Commedia, XLIII, I966, pp. 69-77; Il quarantaquattresimo codice del Convivio, XLIII, I966, pp. 263-264; Quattro stelle non viste mai fuor c'a la prima gente, XLIV, I967, pp. [209]-210; Riflessioni sul testo del Convivio, XLIV, I967, pp. 85-II4; Il verbo 'tralucere' nella «Divina Commedia», XLVII, I970, pp. 5-I4; Nuove proposte per il Convivio, XLVIII, I97I, pp. I2I-I36; Note dantesche (Rime LXXX 21-28; Inf. IX 65, XXVIII 121-122), L, 1973, pp. [97]IO2; Altre note dantesche, LI, I978, pp. [215]-220; Interpretazione e punteggiatura in alcuni passi danteschi, «Studi danteschi», LII, I979-80, pp. [I7I]-I88; Alcuni passi danteschi di interpretazione controversa, I98I, pp. 53-62; Il codice già Phillipps della 'Monarchia', LIII, I98I, pp. 295-398; Strumenti per la misurazione del tempo nei paragoni della terza cantica, I982, pp. I13-120; Osservazioni sull'aspetto e il tempo del verbo nella 'Commedia', LI, I982, p. 92; Passi del Convivio inseriti nell' Ottimo Commento, LIV, I982, pp. 137-I56; Nuovi appunti per un commento linguistico alla Commedia, LV, I983, pp. I5I-I63; La funzione delle fonti e dei luoghi paralleli nella fissazione del testo critico: esperienze di un editore del 'Convivio', LVIII, I986, pp. 239-273; La funzione delle fonti e dei luoghi paralleli nella fissazione del testo critico: esperienze di un editore del Convivio, LVIII, I986, pp. 239-273; Un fracasso di un suon pien di spavento (Inf. IX 65), III, I990, pp. 40-4I), oltre ad alcuni saggi in rivista e in volume (Osservazioni sull'aspetto e il tempo del verbo nella Commedia, in "Studi di Grammatica italiana", I, I97I, pp. 6I-Ioo; Coordinazione di indicativo e congiuntivo in taluni tipi di secondarie presso Dante e Un tipo di consecutive senza antecedente nella Commedia, in "Studi di Grammatica italiana", III, I973, pp. I39-I50; Imperativo in secondaria: 'rifronzire' hapax dantesco?, in "Lingua nostra", XLIII, I982, p. II6; Ancora per l'edizione critica del Convivio, in La Società Dantesca Italiana 1888-1988: Convegno internazionale (Firenze, 24-26 novembre 1988), MilanoNapoli, Ricciardi, I995, pp. 337-56), alle schede nelle Appendici dell'Enciclopedia dantesca riguardanti l'uso del verbo nelle opere di Dante (Tempi dell'indicativo, Congiuntivo, Condizionale, Imperativo, Infinito, Gerundio, Participio, pp. 222-317; Diatesi, Aspetto del verbo, Concordanze, pp. 320-334; Periodo ipotetico, Accusativo e infinito, Consecutio temporum, Perifrasi verbali, Paraipotassi, Anacoluto, pp. 408-444, in Enciclopedia Dantesca, Appendice: biografia, lingua e stile opere, Roma, Istituto della Enciclopedia italiana, 1978) e al già menzionato volume Il verbo nellitaliano antico: ricerche di sintassi (Milano-Napoli, Ricciardi, I964).

La fitta bibliografia appena proposta rappresenta in realtà soltanto un estratto delle numerosissime pubblicazioni di Franca Brambilla Ageno: dalle carte d'Archivio emergono, infatti, moltissime altre testimonianze relative alla sua attività nell'ambito degli studi lessicali.

Nel fascicolo segnato iz88 (Sacchetti I), si trovano alcune schede risalenti alla composizione del saggio Riboboli trecenteschi, uscito negli "Studi di Filologia italiana" del 1952 (pp. 4I3-454) e riguardante molti aspetti lessicali di testi del Trecento. Tra i casi considerati da Franca Brambilla Ageno, si sono voluti prendere in considerazione alcuni esempi relativi ad appellativi dispregiativi riferiti alle donne:

Ciscranna, il - (348): REW 6II, 8009, Burch., p. 6. Scranna è tuttora usato nel senso di 'donna vizza e brutta' o 'scostumata', Fanfani [...]. 
Zambracca (333): 'donna di cattivi costumi': forse da zambra nel significato di 'cesso', Prati, e vi può aver influito baldracca da Baldacco, un luogo in Firenze con osteria di quel nome. Corb., p. 23I: «Per ire dietro alle fanti e alle zambracche e alle vili e alle cattive femmine» ${ }^{\mathrm{IO}}$.

Si tratta, dunque, delle voci ciscranna, 'donna vizza, brutta e scostumata', e zambracca, 'donna di cattivi costumi, cesso'. Per ciscranna, la Ageno si rifa al REW ${ }^{\mathrm{II}}$ e a Pietro Fanfani ${ }^{\mathrm{I2}}$, mentre per zambracca si riporta la definizione di Prati'13, oltre a un esempio dal Corbaccio di Boccaccio. Zambracca, poi, potrebbe derivare da Baldacco, bordello e luogo disonorevole di Firenze, oltre a essere alterazione toscana del nome della città di Bagdad ${ }^{\mathrm{I}}$. Di entrambi questi termini si rintracciano le relative schede tra le carte d'Archivio (fasc i388, u.d. r8b). In particolare, si legga la c. 74, a proposito di zambracca [4]:

\footnotetext{
Zambracca, v. 333 - Fiera I II 2: "Ma di quai ve ne son, ch'han si ritrosa / la fede verso '1 medico, ch'occulti / chiamati i cerretani e le zambracche...". Prati: Per disprezzo, dal secondo significato [di zambra: cesso] forse venne zambracca, "femmina di mondo o vile (Bocc., Med., Grazz.; (spreg.) cameriera (Buon. Il G.)". Zambracca è anche del nap. e zambracca del vic., mentre il venez. à salambraca "cimbraccola". Cimbràccola o cirimbraccola (tosc. volg.) "donna sciatta e bècera" deriva da cimbràccole, "panni di poco valore", cimbràccolo "ciondolo, straccio" [...].
}

Rispetto alla scheda proposta nei Riboboli, nei suoi appunti la Ageno fornisce molte più informazioni sulla voce: si segnalano attestazioni in altri autori (anche non trecenteschi) e si indica la presenza del termine, in altre forme, anche nei dialetti (in napoletano, in vicentino, in veneziano e in toscano). Un'analisi, dunque, molto più ampia, della quale, però, si sceglie di pubblicare nel saggio solo una piccola parte: è dalle carte d'Archivio, quindi, che ancora una volta è possibile accedere all'ampio materiale della studiosa e osservare la complessità del suo lavoro.

In queste poche pagine, dunque, si è cercato di sottolineare alcuni aspetti relativi agli importanti lavori di Franca Brambilla Ageno, una delle studiose più importanti nell'ambito delle ricerche filologiche e linguistiche, spesso non conosciuta al di fuori dell'ambito specialistico. Consultare le carte della Ageno significa osservare da vicino il suo metodo di studio e di lavoro, ma vuol dire anche e soprattutto leggere tra le righe della vita di una donna dal carattere spesso schivo (come spesso molti l'hanno ricordata) di cui oggi siamo in grado di riconoscere la grande eredità.

Note:

I. Ghino Ghinassi, Introduzione, in Franca Brambilla Ageno, Studi lessicali, a cura di Paolo Bongrani, Franca Magnani, Domizia Trolli, Bologna, CLUEB, 200o, p. IX.

2. Carlo Delcorno, Necrologio: Franca Brambilla Ageno, in "Giornale storico della Letteratura italiana", I (I996), p. 3I 8

3. Per un inventario completo del Fondo, si veda Caterina Canneti, Il Fondo Franca Brambilla Ageno all'Accademia della Crusca, in "StEFi - Studi di Erudizione e Filologia", VI (2017), pp. 283-338.

4. Cfr. ivi, p. 284 , n. I.

5. Per le carte di lavoro della Ageno su Franco Sacchetti, si legga Paolo Pellegrini, Tra le carte di Franca Brambilla Ageno: l'edizione delle Opere di Franco Sacchetti, in "Storie e Linguaggi", II (2016), pp. I45-58.

6. Si guardi a pubblicazioni quali: Per una nuova edizione della Battaglia del Sacchetti, «Studi di Filologia 
italiana», XI (I953), pp. 245-206; Una forma friulana in una novella del Sacchetti, in "Tesaur", IX (I957), pp. I2-I3; rec. a Franco Sacchetti, Opere, a cura di Aldo Borlenghi, Milano, Rizzoli, I957, in "Giornale storico della Letteratura italiana", CXXXIV (I957), pp. 368-92; A proposito di una fonte sacchettiana, in "Giornale storico della letteratura italiana", CXXXVII (I960), pp. 204-2I7; Rime estravaganti del Sacchetti, in "Lettere italiane", XIII (I96I), pp. I-I9.

7. Franco Sacchetti, Il libro delle Rime, a cura di Franca Brambilla Ageno, Firenze, Olschki-University of Western Australia Press, I990.

8. Emidio De Felice - Aldo Duro, Vocabolario italiano, Torino, Società editrice internazionale, Palermo, Palumbo, i993.

9. Cfr. Andrea Canova (a cura di), Tra filologia e storia della lingua italiana. Per Franca Brambilla Ageno, Roma, Edizioni di storia e letteratura, p. 82.

Io. Franca Ageno, Riboboli trecenteschi, in "Studi di Filologia italiana", X (I952), pp. 449-50.

II. W. Meyer-Lübke, Romanisches Etymologisches Wörterbuch, Heidelberg, I9II, Carl Winter's, Universitätsbuchhandlung.

I2. Cfr. Vocabolario della lingua italiana, Firenze, Le Monnier, I855 (e numerose successive edizioni) e Vocabolario dell'uso toscano, 2 voll., Firenze, G. Barbera, I863.

I3. Cfr. Angelico Prati, Vocabolario etimologico italiano, Milano, Garzanti, I95I.

I4. Cfr. Vocabolario Treccani on-line, s.v. baldacco (http://www.treccani.it/vocabolario/baldacco/).

Immagini:

[I]
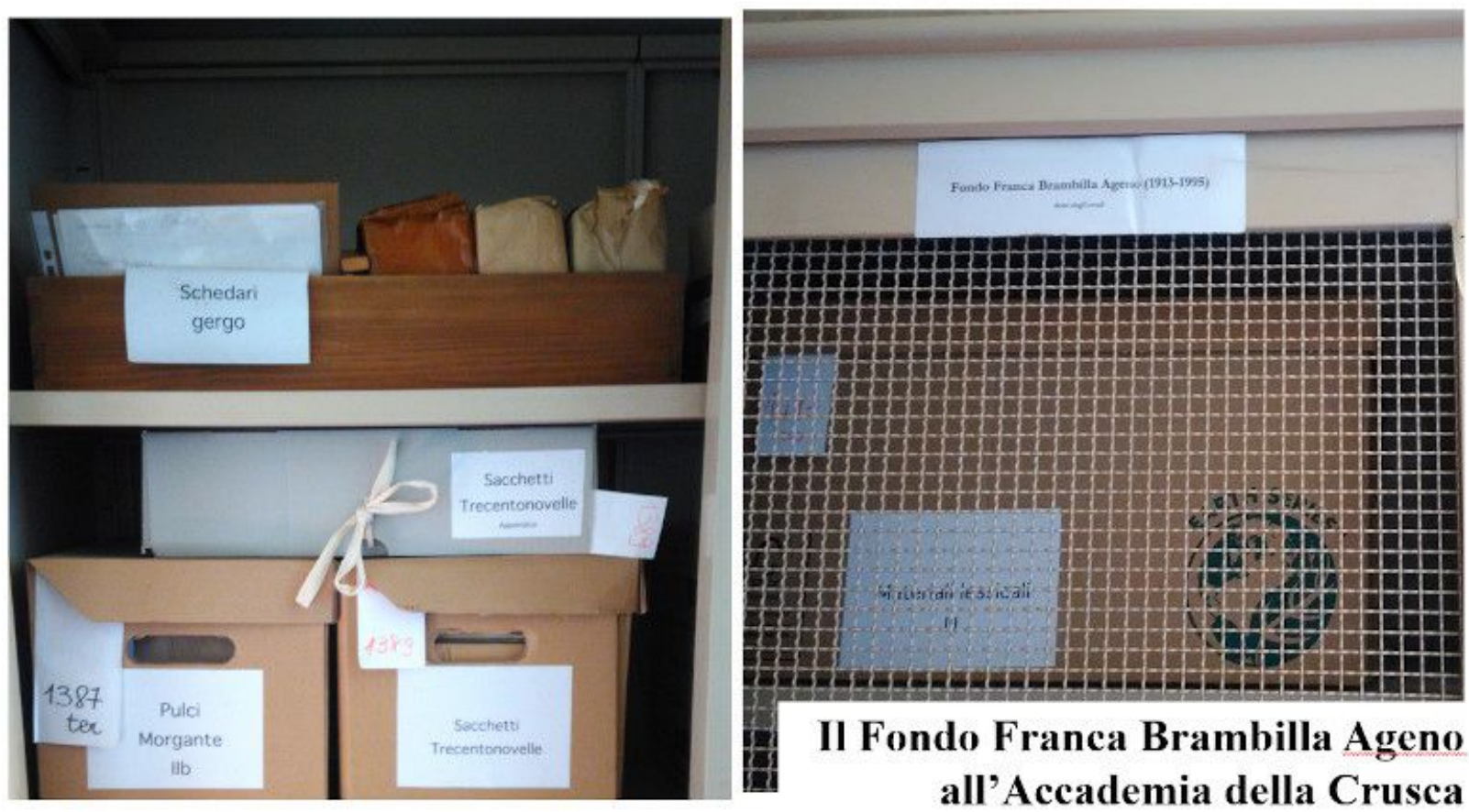
[2]
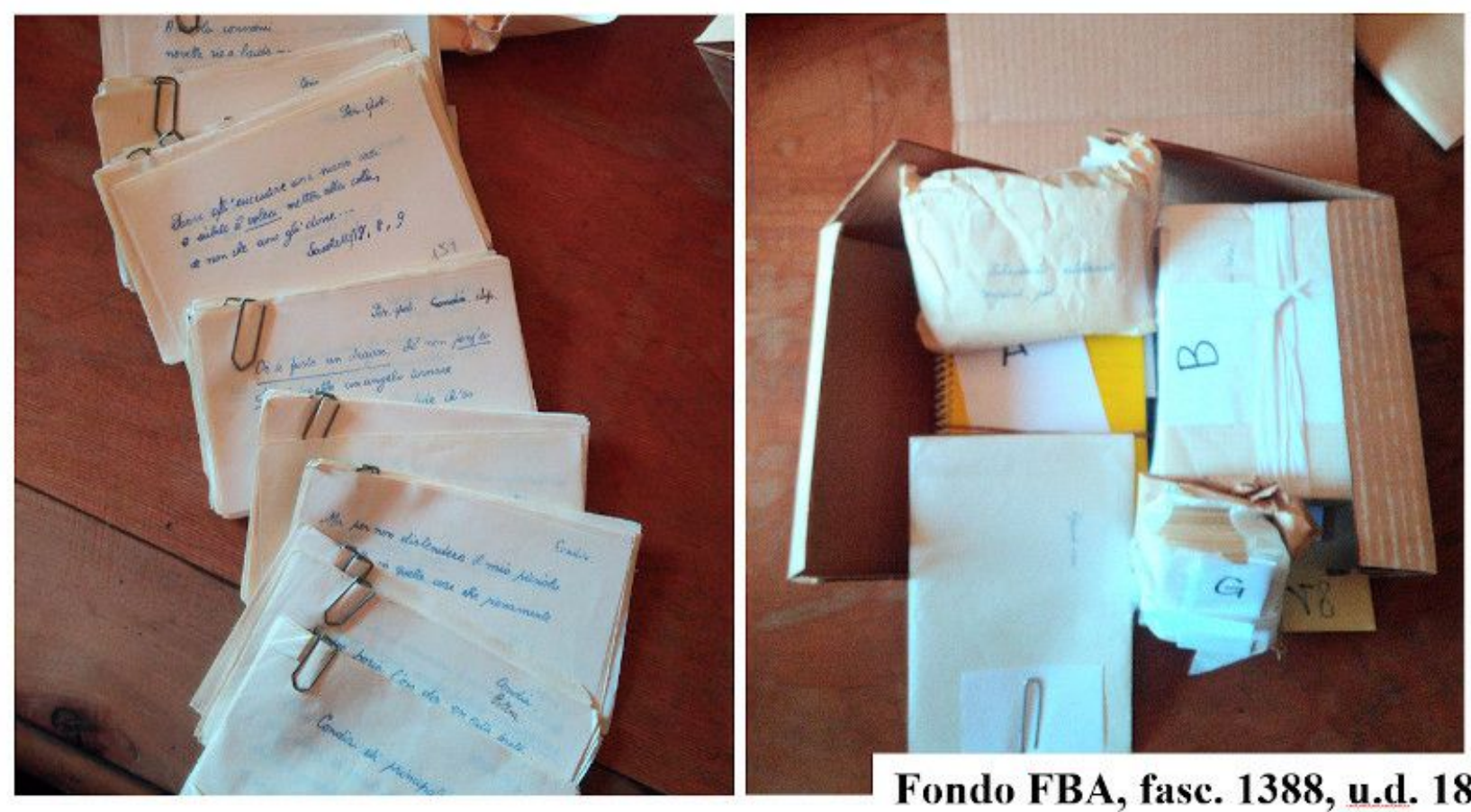

Fondo FBA, fasc. 1388, u.d. 18

[3]
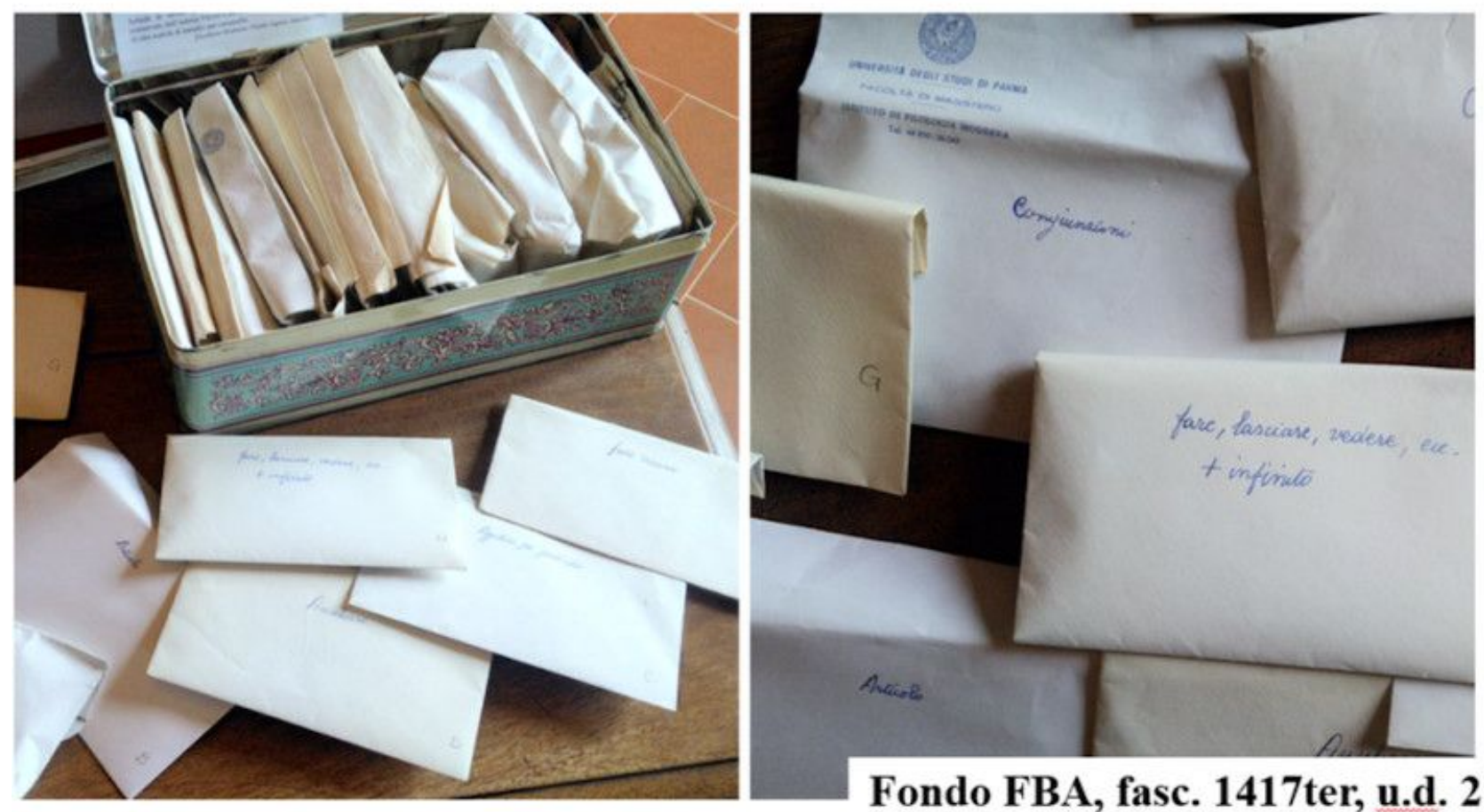

Fondo FBA, fasc. 1417ter, u.d. 2 


\section{$[4]$}

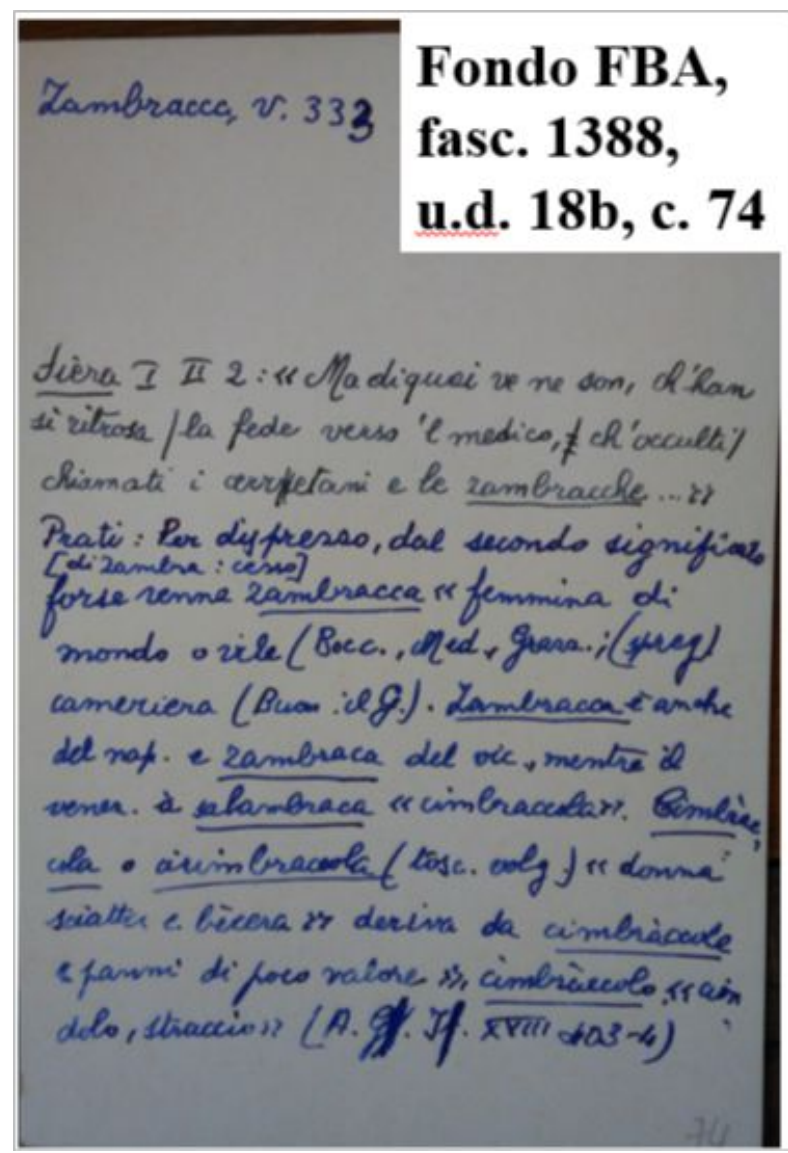

\section{Cita come:}

Caterina Canneti, Ritagli di lingua: uno sguardo sulle carte linguistiche di Franca Brambilla Ageno all'Accademia della Crusca, "Italiano digitale", 2019, XI, 2019/4 (ottobre-dicembre) DOI: $10.35948 / 2532-9006 / 2020.3164$

Copyright 2019 Accademia della Crusca Pubblicato con licenza creative commons CC BY-NC-ND 